\title{
Evaluation of Stability and Antibacterial Activity of Various Concentrations of Triple Antibiotic Paste against Enterococcus faecalis: An in vitro Study
}

${ }^{1}$ Divya Subramanyam, ${ }^{2}$ Sujatha Somasundaram

\begin{abstract}
Aim: The aim of this study is to evaluate the stability and antibacterial effect of various concentrations of triple antibiotic paste (TAP) against Enterococcus faecalis with antibiotic susceptibility testing.
\end{abstract}

Materials and methods: The stability and antibacterial effect of TAP was tested with antibiotic susceptibility test against E. faecalis by using Agar disk diffusion method over a period of $1,3,7,14$, and 21 days. The samples were divided into three groups: group I (1\% TAP), group II ( $2 \%$ TAP), group III (3\% TAP), and group IV (chlorhexidine as control). The zones of inhibition were measured after 24 hours and were recorded in millimeters and the same procedure was repeated again after $3,7,14$, and 21 days.

Results: Higher concentration of $3 \%$ TAP showed superior antibacterial effect against $E$. faecalis compared with $1 \%$ TAP, $2 \%$ TAP, and control group (chlorhexidine). The antibacterial efficacy increased more rapidly after 21 days when compared with 24 hours, 3 days, and 7 days.

Conclusion: Under specified limitations of this in vitro study, higher concentration of TAP can be used as an effective intracanal medicament against $E$. faecalis. It is clearly experimented that longer duration of application of intracanal medicament increases its antibacterial effect against $E$. faecalis.

Clinical significance: Triple antibiotic paste can be prepared, stabilized, and used for a period of 21 days.

Keywords: Enterococcus faecalis, Stability, Triple antibiotic paste.

How to cite this article: Subramanyam D, Somasundaram S. Evaluation of Stability and Antibacterial Activity of Various Concentrations of Triple Antibiotic Paste against Enterococcus faecalis: An in vitro Study. World J Dent 2017;8(5):403-406.

Source of support: Nil

Conflict of interest: None

\section{INTRODUCTION}

Periapical root canal infection in primary teeth is a common sequelae of early childhood caries in primary

1,2Department of Pedodontics, Saveetha Dental College Chennai, Tamil Nadu, India

Corresponding Author: Divya Subramanyam, No 20, School Road, Venkateshwara Nagar East Phase, Kodungaiyur, Chennai 600118, Tamil Nadu, India, Phone: +919003226380, e-mail: smiley.divya24@gmail.com dentition. ${ }^{1}$ The accomplishment of a successful root canal therapy is to eliminate the periapical microbiota, which are potential resource of recurrent infection, preventing the failure of endodontic treatment. Due to the presence of numerous lateral canals and apical ramifications in the primary molar, complete mechanical debridement of necrotic tissue is entirely hindered and pose many difficulties. ${ }^{2,3}$ Bacteria which persists in the root canals even after mechanical debridement is the primary causative factor responsible for the development and progress of periradicular inflammatory lesions. ${ }^{4}$ Control of periapical infection is necessary to prevent the infection spreading into the underlying permanent tooth bud which lies in close proximity to the wide furcated roots of the deciduous teeth. ${ }^{5}$ When failure of endodontic treatment occurs due to persistent bacterial infection in the root canal system, it is called as secondary infection. ${ }^{6}$ The bacteria associated with periapical infection in primary teeth is polymicrobial in nature with a combination of Grampositive, Gram-negative aerobic and anaerobic organisms inhabiting the root canals, but studies have reported the maximum predominance of anaerobic microorganisms in the infected root canals. ${ }^{\text {? }}$

Enterococcus faecalis is a facultative anaerobe highly resistant to chemicomechanical preparation done during root canal therapy, which is responsible for persistent secondary root canal infections as the microbe has an inherent ability to survive in an oxygen-free environment. ${ }^{8}$ Many studies have reported that there is a higher prevalence of E. faecalis in persistent root canal infections., ${ }^{9,10}$ Enterococcus has been isolated from $47 \%$ of endodontically treated root canals in which treatment has failed. ${ }^{11}$ Effective antimicrobial field is achieved in the root canals, both by thorough mechanical debridement and placement of antimicrobial agent to destroy any further existing microorganisms ${ }^{12}$ (Fuks et al, 2000). Over many years, various materials have been used as an intracanal medicament in primary teeth like formocresol, glutaraldehyde, calcium hydroxide, chlorhexidine gel, corticosteroids, and certain antibiotics. Among the numerous properties accounted for in an ideal intracanal medicament, stability, biocompatibility, and antibacterial action are the most important factors to prevent multiplication of certain microorganisms between intraappointment visits. ${ }^{13}$ 
A single antibiotic does not provide adequate sterilization and eliminate the microbes present in the root canals. Over the past decades, the resistance of microorganisms to antibiotics has raised due to regular use of antibiotics for various infections. ${ }^{11}$ Therefore, to eliminate the variety of microbes present in the complex root canal system, a mixture of antibiotics like ciprofloxacin, metronidazole, and doxycycline introduced by Hoshino et $\mathrm{al}^{14}$ has been used as nonsurgical endodontic therapy to eradicate the resistant microbes. A periapical lesion responds to nonsurgical endodontic therapy because of the complete disinfection of the root canal system with the help of intracanal medicament. Antibiotics are essential for inhibition of bacterial infections. The Cariology Research Unit of the Niigata University School of Dentistry introduced the concept of "Lesion sterilization and tissue repair therapy (LSTR)". Nonsurgical endodontic therapy, such as local application of antibiotics in the form of "LSTR" has been on the rise in recent years to avoid the possible side effects of systemic antibiotics. ${ }^{14}$ Literature has shown that a mixture of ciprofloxacin, metronidazole, and doxycycline produces sterilization of root canal dentin by penetrating and completely eradicating the resistant bacteria. Various studies evaluated the antibacterial effectiveness of TAP against $E$. faecalis but there is a lacunae of research regarding the stability of TAP at different concentrations against E. faecalis over a period of 3 days. This study aims to determine the stability of TAP by evaluating the antibacterial effect of various concentrations against E. faecalis using antibiotic susceptibility testing.

\section{MATERIALS AND METHODS}

The study approval was obtained from the Institutional Review Board, Saveetha Dental College. Triple antibiotic paste made of metronidazole, ciprofloxacin, and doxycycline (1:1:1) was prepared by using sterile mortar and pestle at different concentrations using propylene glycol as a carrier in the ratio of 1:1 and stored under sterile conditions. The antibacterial effect of TAP was tested by antimicrobial susceptibility testing against $E$. faecalis by using Agar disk diffusion method over a period of 24 hours and after 3, 7, 14, and 21 days respectively. The samples were divided into three groups: group I ( $1 \%$ TAP), group II ( $2 \%$ TAP), group II ( $3 \%$ TAP), and group IV (chlorhexidine as control). Mueller-Hinton agar plates were used and inoculated with E. faecalis, and sterile discs with different concentrations of TAP were placed and incubated for 24 hours at $37^{\circ} \mathrm{C} .{ }^{15}$ The zones of inhibition were measured after 24 hours and were recorded in millimeters and the same procedure was repeated again after 3, 7, 14, and 21 days. The results were analyzed statistically by Kruskal-Wallis test at 5\% significance level. The sterile discs (5 $\mathrm{mm}$ diameter) were dipped aseptically in
Table 1: Comparison of zone of inhibition of $1 \%, 2 \%, 3 \%$ TAP and chlorhexidine

\begin{tabular}{llllll}
\hline & \multicolumn{5}{c}{ Zone of inhibition of E. faecalis $(\mathrm{mm})$} \\
\cline { 2 - 6 } Sample & 24 hours & 3 days & 7 days & 14 days & 21 days \\
\hline $1 \%$ TAP & 34 & 30 & 36 & 41 & 27 \\
$2 \%$ TAP & 40 & 34 & 40 & 46 & 29 \\
$3 \%$ TAP & 43 & 36 & 46 & 49 & 32 \\
Chlorhexidine & 17 & 20 & 27 & 20 & 15 \\
\hline
\end{tabular}

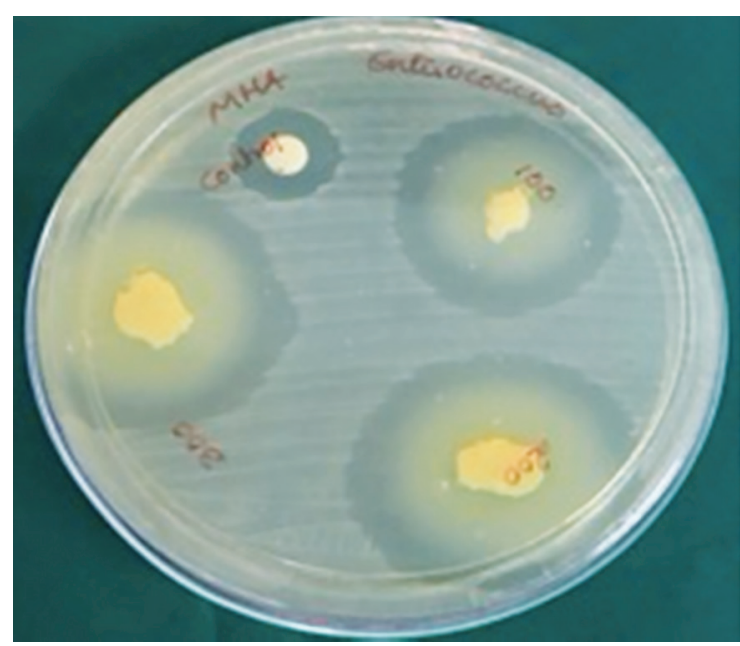

Fig. 1: Zone of bacterial inhibition against $E$. faecalis after 21 days

different extracts for 1 minute and placed over nutrient agar plates seeded with bacterial culture. The plates were left at ambient temperature for 15 minutes and then incubated at $37^{\circ} \mathrm{C}$ for 16 hours and observed for zone of inhibition.

\section{RESULTS}

The inhibitory zones at various concentrations of TAP and chlorhexidine against E. faecalis are shown in Table 1 and Figure 1. From the disk diffusion method, it was observed that the zone of inhibition for group 1 (1\% TAP) was 34, 30, 36, 41, and $27 \mathrm{~mm}$ after 24 hours, 3 days, 7 days, 14 days, and 21 days respectively. Group II ( $2 \%$ TAP) was $40,34,40,46$, and $29 \mathrm{~mm}$ at 24 hours, 3 days, 7 days, 14 days, and 21 days respectively, and group III ( $3 \%$ TAP) was 43, $36,46,49$, and $32 \mathrm{~mm}$ at 24 hours, 3 days, 7 days, 14 days, and 21 days respectively. Group IV (chlorhexidine) showed a zone of inhibition of 17, 20,27, 20, and $15 \mathrm{~mm}$ at 24 hours, 3 days, 7 days, 14 days, and 21 days respectively. The greater antibacterial effect of TAP was seen at higher concentrations (Table 1). The antibacterial effect of TAP decreased after 3 days but was seen to increase over a period of 21 days. But chlorhexidine showed a greater zone of inhibition after $3,7,21$ days compared with 24 hours against $E$. faecalis.

\section{DISCUSSION}

The important objective of endodontic therapy is to provide complete disinfection of the root canal walls 
to prevent recurrent infection. ${ }^{16}$ The complex nature of the root canals leaves certain microbes untouched by mechanical instrumentation and irrigation. The facultative anaerobe like E. faecalis was used as a test organism in this study as it is most predominantly seen microbiota in the root canals. Enterococcus faecalis is the main organism harboring in the root canal leading to persistent endodontic infection. ${ }^{17}$ Among various facultative anaerobes present in the root canals, E. faecalis has the ability to survive in an ecologically changing environment, which imposes a greater challenge to eradicate it from the root canals. ${ }^{18}$ It has the capacity to survive without the support of other microbes in anaerobic conditions. It has been reported that $E$. faecalis is resistant to calcium hydroxide. ${ }^{19}$ Turk et $\mathrm{al}^{20}$ reported that chlorhexidine along with calcium hydroxide showed better antibacterial effect compared with calcium hydroxide used alone.

So, in this study chlorhexidine was used as a control as it possesses many properties of an ideal intracanal medicament, such as superior antimicrobial activity, substantivity, and lower cytotoxicity. The clinical effectiveness of chlorhexidine compared with other medicaments is due to its capacity to inhibit matrix metalloproteinase. ${ }^{21}$ It removes the smear layer produced during mechanical instrumentation. ${ }^{22,23}$ Chlorhexidine has better antibacterial property compared with $\mathrm{Ca}(\mathrm{OH})_{2}$ against resistant bacteria present in the root canal. ${ }^{24}$ The use of intracanal medicaments is important for adequate disinfection. This study evaluated the antibacterial efficacy of TAP used at various concentrations over the period of 1 and 3 days to test its stability as an intracanal medicament by disk diffusion method. American Type Culture Collection 29212 strains of E. faecalis were used in this in vitro study. Both agar diffusion method and agar well method were used to evaluate antimicrobial susceptibility. Agar disk diffusion method was used in this study as it is a proven method in research for evaluating the antibacterial property. ${ }^{25,26}$ It has less technique sensitivity and also has the advantage of detecting the antimicrobial resistance of medicaments using sterile filter paper discs without altering the chemical properties of medicaments. ${ }^{27}$ Mueller-Hinton agar was used as it is the selective medium for growth of all fastidious organisms.

The results from this study showed that the most effective medicament against $E$. faecalis was 3\% TAP compared with other groups ( $2 \%$ TAP > $1 \%$ TAP > chlorhexidine); however, all the concentration of TAP could completely disinfect the $E$. faecalis. This was in agreement to a study done by Mozayeni et $\mathrm{al}^{28}$ who observed that chlorhexidine and TAP were effective against $E$. faecalis compared with calcium hydroxide, but the effectiveness of TAP was superior compared with chlorhexidine gel. Therefore, this study proves that higher the concentration of antibiotics used, higher the antibacterial effectiveness. Contradictory to this present study, Reyhani et $\mathrm{al}^{29}$ observed that the antibacterial effect when used for 1 week was similar to that of 4 weeks. In the present study, the effectiveness of chlorhexidine increased after 3 and 7 days, which explains its substantivity. It has been reported that the action of chlorhexidine increases from up to 72 hours to almost 21 days. ${ }^{30}$ In future, more in vivo and in vitro studies are advocated to determine the stability and antibacterial effect of TAP under proper oral scenario over longer period of time to confirm the findings obtained from this study.

\section{CONCLUSION}

From this study, it is observed that higher concentrations of TAP can effectively eliminate E. faecalis from the root canals compared with chlorhexidine when administered over a longer period of time. Triple antibiotic paste was found to have increased stability and can be stored for a period of 21 days.

\section{CLINICAL SIGNIFICANCE}

Triple antibiotic paste mixture can be stabilized and used for a period of 21 days.

\section{REFERENCES}

1. Reddy S, Ramakrishna Y. Evaluation of antimicrobial efficacy of various root canal filling materials used in primary teeth: a microbiological study. J Clin Pediatr Dent 2007 Spring; 31(3):193-198.

2. Baker BCW, Parsons KC, Mills PR, Williams GL. Anatomy of root canals. IV deciduous teeth. Aust Dent J 1975 Apr;20(2): 101-106.

3. Zurcher, E. The anatomy of the root canals of the teeth of the permanent and deciduous dentitions and of the first permanent molars. New York (NY): William Wood and Co.; 1925.

4. Bystrom A, Claesson R, Sundqvist G. The antibacterial effect of camphorated paramonochlorophenol, camphorated phenol and calcium hydroxide in the treatment of infected root canals. Endod Dent Traumatol 1985 Oct;1(5):170-175.

5. Haapasalo M, Udnaes T, Endal U. Persistent, recurrent, and acquired infection of the root canal system post-treatment. Endodontic Topics 2003 Nov;6(1):29-56.

6. Kantz WE, Henry CA. Isolation and classification of anaerobic bacteria from intact pulp chambers of non-vital teeth in man. Arch Oral Biol 1974 Jan;19(1):91-96.

7. Pazelli LC, de Freitas AC, Ito IY, de Souza-Gugelmin MC, Medeiros AS, Nelson-Filho P. Prevalence of microorganisms in root canals of human deciduous teeth with necrotic pulp and chronic periapical lesions. Pesqui Odontol Bras 2003 Dec;17(4):367-371.

8. Shailaja S, Suresh BS. Endodontic micro flora-a review. J Oral Health Com Dent 2014 Sep;8(3):160-165.

9. Möller AJ. Microbiological examination of root canals and periapical tissues of human teeth. Methodological studies. Odontol Tidskr 1966 Dec;74(5):(Suppl):1-380. 
10. Peciuliene V, Balciuniene I, Eriksen HM, Haapasalo M. Isolation of Enterococcus faecalis in previously root-filled canals in a Lithuanian population. J Endod 2000 Oct;26(10):593-595.

11. Pinheiro ET, Gomes BP, Ferraz CCR, Sousa ELR, Teixeira FB, Souza-Filho FJ. Microorganisms from canals of root-filled teeth with periapical lesions. Int Endod J 2003 Jan;36(1):1-11.

12. Deshpande A, Sudani U. Intracanal medicament in pediatric endodontics: a literature review. J Adv Med Dent Sci Res 2015 Jan;3(2):63-68.

13. Vijayaraghavan R, Mathian VM, Sundaram AM, Karunakaran R, Vinodh S. Triple antibiotic paste in root canal therapy. J Pharm Bioallied Sci 2012 Aug;4(Suppl 2):S230-S233.

14. Mandwe Ashish P, Patil Pravin G, Sheety Heerish K, Shetty Vathsalya K, Pimpale Sandeep K. Role of triple antibiotic paste in revascularization and endodontics-a review Int J Contemp Med Res 2015 Nov;2(2):5.

15. Balouiri M, Sadiki M, Ibnsouda SK. Methods for in vitro evaluating antimicrobial activity: a review. J Pharm Anal 2016 Apr;6(2):71-79.

16. Sjogren U, Figdor D, Persson S, Sundqvist G. Influence of infection at the time of root filling on the outcome of endodontic treatment of teeth with apical periodontitis. Int Endod J 1997 Sep;30(5):297-306.

17. Narayanan LL, Vaishnavi C. Endodontic microbiology. J Conserv Dent 2010 Oct-Dec;13(4):233-239.

18. Williams JM, Trope M, Caplan DJ, Shugars DC. Detection and quantitation of E. faecalis by real-time PCR (qPCR), reverse transcription-PCR (RT-PCR), and cultivation during endodontic treatment. J Endod 2006 Aug;32(8):715-721.

19. Evans MD, Baumgartner JC, Khemaleelakul SU, Xia T. Efficacy of calcium hydroxide-chlorhexidine paste as an intra canal medication in bovine dentin. J Endod 2003 May;29(5):338-339.

20. Turk BT, Sen BH, Ozturk T. In vitro antimicrobial activity of calcium hydroxide mixed with different vehicles against Enterococcus faecalis and Candida albicans. Oral Surg Oral Med Oral Pathol Oral Radiol Endod 2009 Aug;108(2):297-301.
21. Gomes BP, Vianna ME, Zaia AA, Almeida JF, Souza-Filho FJ, Ferraz CC. Chlorhexidine in endodontics. Braz Dent J 2013 Mar;24(2):89-102.

22. Ferraz CC, Gomes BP, Zaia AA, Teixeira FB, Souza-Filho FJ. In vitro assessment of the antimicrobial action and the mechanical ability of chlorhexidine gel as an endodontic irrigant. J Endod 2001 Jul;27(7):452-455.

23. Pinheiro ET, Gomes BP, Ferraz CC, Teixeira FB, Zaia AA, Souza Filho FJ. Evaluation of root canal microorganisms isolated from teeth with endodontic failure and their antimicrobial susceptibility. Oral Microbiol Immunol 2003 Apr;18(2):100-103.

24. Siqueira JF Jr, Batista MM, Fraga RC, de Uzeda M. Antibacterial effects of endodontic irrigants on black-pigmented gram-negative anaerobes and facultative bacteria. J Endod 1998 Jun;24(6):414-416.

25. Poggio C, Colombo M, Scribante A, Sforza D, Bianchi S. In vitro antibacterial activity of different endodontic irrigants. Dent Traumatol 2012 Jun;28(3):205-209.

26. Carson KR, Goodell GG, McClanahan SB. Comparison of the antimicrobial activity of six irrigants on primary endodontic pathogens. J Endod 2005 Jun;31(6):471-473.

27. Mohammadi Z, Shahriari S. Residual antibacterial activity of chlorhexidine and MTAD in human root dentin in vitro. J Oral Sci 2008 Mar;50(1):63-67.

28. Mozayeni MA, Haeri A, Dianat O, Jafari AR. Antimicrobial effects of four intracanal medicaments on Enterococcus faecalis: an in vitro study. Iran Endod J 2014 Summer;9(3):195-198.

29. Reyhani FM, Rahimi S, Fathi Z, Shakouie S, Milani AS, Barhaghi MHS, Shokri J. Evaluation of antimicrobial effects of different concentrations of triple antibiotic paste on mature biofilm of Enterococcus faecalis. J Dent Res Dent Clin Dent Prospects 2015 Summer;9(3):138-143.

30. Komorowski R, Grad H, Wu XY, Friedman S. Antimicrobial substantivity of chlorhexidine treated bovine root dentin. J Endod 2000 Jun;26(6):315-317. 